\title{
Why Hacking is wrong about human kinds.
}

\begin{abstract}
'Human kind' is a term introduced by Ian Hacking to refer to the kinds of people-child abusers, pregnant teenagers, the unemployed-studied by the human sciences. Hacking argues that classifying and describing human kinds results in feedback, which alters the very kinds under study. This feedback results in human kinds having histories totally unlike those of natural kinds (such as copper, tigers and dandelions) leading Hacking to conclude that human kinds are radically unlike natural kinds. Here I argue that Hacking's argument fails and that he has not demonstrated that human kinds cannot be natural kinds.
\end{abstract}

1. Natural kinds.

2. Hacking's feedback mechanisms.

2.1 Cultural feedback.

2.2 Conceptual feedback

Child abusers, pregnant teenagers and the unemployed are examples of what Ian Hacking terms 
'human kinds'. They are kinds of people studied by the human sciences. 'Human kind' is a term chosen by Hacking to contrast with 'natural kind'. Natural kinds are the kinds dealt with by the natural sciences. Traditional examples include gold, electrons, and tigers.

Hacking's central claim is that human kinds and natural kinds are fundamentally distinct ([1986], [1988], [1992], [1995a], [1995b]). If Hacking is right, this has profound implications for the question of whether the human sciences are fundamentally like, or unlike, the natural sciences. Natural kinds are often considered to feature in natural laws. Many have also considered natural kinds to play a key role in explanations and in supporting inductive inferences. As such, if human kinds cannot be natural kinds, this may indicate that laws and explanations will take different forms in the natural and human sciences.

Since developing the arguments that are discussed here, Hacking seems to have changed tack and, although he does not give reasons for rejecting his earlier work, Chapter Four of his The Social Construction of What? ([2000]) discusses the possibility that at least some human kinds (called 'interactive kinds' in this book) are also natural kinds. Hacking discusses autism as a possible example. Here I am only concerned with Hacking's earlier work. His argument that human kinds cannot be natural kinds has been influential, so far as I know it as yet stands unrefuted and, if sound, it has radical implications for the philosophy of the human sciences.

Hacking's argument that human kinds cannot be natural kinds rests on the claim that classifying and describing human kinds results in feedback which alters the very kinds under study. In a nutshell, homosexuals respond to being studied, spiders and copper do not. The resulting feedback means that human kinds have histories totally unlike the histories of natural kinds. Thus, Hacking concludes, human kinds cannot be natural kinds.

In this paper I argue that Hacking is wrong. I claim that he has failed to show that human kinds cannot be natural kinds. The feedback that Hacking claims makes human kinds so very different from natural kinds is supposed to operate at two levels, a cultural level and a conceptual level. I will examine each type of feedback in turn, and show that in so far as feedback occurs it is compatible with human kinds being natural kinds. Before going on to outline and assess Hacking's arguments, however, it will be helpful to first get clearer about the nature of natural kinds.

\section{Natural Kinds}


Hacking claims that human kinds cannot be natural kinds, but what exactly are natural kinds? Unfortunately, a straightforward answer cannot be given here. There are multiple accounts of natural kinds, and all are controversial. Essentialists claim that all members of a natural kind share some essential property (see for example, Wilkerson [1995]). These essential properties fulfil two roles. First, the class of entities possessing the essential property delineates the membership of the kind-to belong to the natural kind 'gold' it is necessary and sufficient to have an atomic number of 79 . Second, the essential property largely determines the other properties possessed by members of the kind-it is a lawful consequence of having an atomic number of 79 that a piece of material will be metal, will conduct electricity, will be solid at $20^{\circ} \mathrm{C}$, will be largely inert, and so on.

A major difficulty for an essentialist account of natural kinds is that for biological species, which are traditionally considered amongst the best examples of natural kinds, no plausible candidates for the essences can be found. As John Dupré ([1981], pp.84-5) has pointed out there are reasons for thinking that often there will be no one genetic property or set of properties shared by all members of a species. Most importantly, evolutionary theory suggests that it will be beneficial for there to be variation in the genes possessed by members of a species as this will enable the species to adapt quickly when the environment changes. The existence of genetic diseases gives another reason for thinking that the genetic properties of members of a kind will vary.

There are two possible ways of responding to such concerns. Terence Wilkerson ([1995]) chooses to stick with essentialism. He argues that if biological species lack essential properties then this only goes to show that they are not natural kinds. If such an option is taken then it becomes hard to see the motivation behind Hacking's argument. Someone who thinks that biological species are not natural kinds will almost certainly already think that human kinds cannot be natural kinds either. To such people Hacking will seem to be arguing for the obvious.

Alternatively, one may claim that biological species are paradigmatic examples of natural kinds, and that if essentialist accounts of natural kinds cannot accommodate them, essentialism must be rejected. This is the route taken by Dupré himself ([1981], [1993]), and by Richard Boyd ([1991]), amongst others. A variety of non-essentialist accounts of natural kinds, designed to deal with biological species, have now been proposed. I will outline three of these non-essentialist accounts of natural kinds below, in order to give the reader some flavour of current debates. If a non- 
essentialist account of natural kinds is adopted then it is a serious possibility that human kinds might be natural kinds, and Hacking's argument will be of interest.

John Dupré ([1981], [1993]) argues for an account he calls 'promiscuous realism'. He asks us to imagine the individual entities of some domain (he considers biological organisms but his ideas can be generalised) mapped out on a multidimensional quality space. Dupré claims that in such a map we would find numerous clusters corresponding to groups of similar entities. In many cases the clusters will not be discrete, but will be messy and hard to make out. Some clusters will correspond to traditional natural kinds. At different levels of resolution other clusters might be discerned-as well as a cluster that corresponds to dogs, there will be finer clusters corresponding to dog-breeds, and, at a finer level still, to particular strains of pedigree dogs. Different clusters can also be generated by restricting our attention to particular dimensions of the map. If we restrict our attention to the dimensions that code for nutritional value, for example, we will find a cluster of things that are poisonous to humans. Dupré's account is realist because the clusters in quality space reflect the real structure of nature. It is promiscuous because there will be many different clusters on which we could choose to focus.

Other non-essentialist accounts have been proposed by Richard Boyd and by Ruth Millikan. Like Dupré, Boyd ([1991]) argues that members of a kind possess a cluster of regularly cooccurring properties. Unlike Dupré, he stresses that this is for a reason, there is some 'homeostatic' mechanism that makes it the case that these properties re-occur. Boyd's account works well for biological species. Members of a species possess clusters of co-occurring properties and this is as a result of homeostatic mechanisms, such as gene flow between the organisms and pressures that arise from the fact that all members of the species must survive in similar environments. Although he doesn't go into details, Boyd suggests that his account can also accommodate human kinds (although he calls these 'social kinds').

Central to Millikan's ([1997]) account is the idea that species can be considered to be individuals, a thesis originally proposed by M. Ghiselin ([1974]) and D. Hull ([1976]). Like other individuals, species have histories; they develop and eventually die. Unlike normal individuals, species can also be considered as classes of individuals and can be spatially dispersed. Millikan uses the idea that species are individuals to explain how it is possible to run inductive inference over the members of a species. She argues that we can make inductive inferences over the members of a 
species in much the same way that we can make inductive inferences over the temporal parts of an ordinary individual. If someone could speak Turkish yesterday it is a good bet that he can speak it today, and it will be causally because he could speak it yesterday that he can speak it today. Similarly the reasons why one can run inductions over the members of a species are causal, historical reasons. It is because members of a species share common ancestors and live in the same niche that they are similar.

Here I will not attempt to determine which, if any, of the above accounts is correct. As the debates over natural kinds have been intense and ongoing, it is unlikely that any particular account of natural kinds will become generally accepted in the near future. Given this uncertainty concerning the nature of natural kinds, is there any way of determining whether human kinds are natural kinds? Don't we need to get straight about natural kinds before we can decide whether human kinds are distinct? Fortunately for this paper I think that there is a means by which we can sensibly ask whether human kinds are natural kinds in the absence of an agreed account of natural kinds. So long as we reject Wilkerson's view (and, as mentioned earlier, unless we do this Hacking's argument is of little interest) we can point to some paradigmatic examples of natural kinds-chemical elements, fundamental particles, and biological species. As such we can ask whether human kinds are distinct from natural kinds via considering whether human kinds are fundamentally like or unlike kinds such as chemical elements, fundamental particles and biological species.

This approach ties in with what Hacking himself has to say about natural kinds. Hacking holds that nature has kinds, but tends to avoid being drawn into metaphysical disputes about their [2] character. He sees himself as part of a tradition of moderate believers in kinds that includes Mill, Venn, Russell, Price and Quine (Hacking [1992], p.185), but says little more than this. Thinkers in this tradition hold that members of a kind are similar to each other, although they disagree on the extent to which entities have to be similar to count as being members of a kind. Quine ([1969], p.234), for example, speaks of entities being of a kind if they share even one property. At the other extreme, Mill ([1973], p.123) holds that members of a natural kind have 'unknown multitudes of properties in common'.

Rather than providing a detailed account of natural kinds, Hacking tends to illustrate what he talking about by pointing at examples. He lists quarks, cystic fibrosis, mud, the common cold, and sunsets (Hacking [1995b], p.352). This list indicates that Hacking's understanding of 'natural kind' 
is more liberal than that of many theorists. He seems happy to apply the term 'natural kind' to any kinds that we can distinguish in nature. In keeping with tradition, however, Hacking thinks that there will be laws that govern the behaviour of natural kinds. Laws ensure that quarks will behave in quarky ways; Hacking thinks there are also laws that ensure that mud will behave in mud-like ways.

Whether Hacking would be better off restricting his use of the term 'natural kind' to traditional examples (lions and quarks, but not sunsets) is a point that will not be discussed here. However this point should be decided, Hacking accepts that biological species and chemical elements count as natural kinds. In assessing his claim that human kinds cannot be natural kinds, I will consider biological species and chemical elements to be paradigmatic examples of natural kinds. Hacking will be happy with these choices, and they have the advantage of also being acceptable to most theorists writing on natural kinds.

Hacking argues that our classificatory practices can affect human kinds in ways that they cannot affect natural kinds. As a consequence he claims that human kinds cannot be natural kinds. I will assess this claim in the absence of an agreed account of natural kinds via considering whether it is true that the feedback mechanisms Hacking discusses affect human kinds but not kinds of chemical element and biological species. This approach should be acceptable to all but Wilkersonstyle essentialists. Wilkerson-style essentialists will think it obvious that human kinds cannot be natural kinds, and so will not be interested in the debate here in any case.

\section{Hacking's Feedback Mechanisms}

\subsection{Cultural Feedback}

Feedback at the cultural level is dependent on the description of a kind of person entering popular culture. Often human kind terms carry heavy moral overtones; consider for example, 'sexual pervert', 'alcoholic', and 'normal'. Being classified in a certain way may also carry institutionalised benefits or costs. Thus people are motivated to attempt to alter the ways in which they are classified and, as their behaviour changes, so do the kinds under study. Consider, for example, the kind 'obese person'. Attitudes towards obesity result in new human kinds, such as 'people with stapled 
stomachs', coming into existence. The characteristics of both obese and non-obese people are also affected by attitudes towards obesity. When obesity becomes stigmatised obese people will tend to become socially isolated and unhappy, while non-obese people will start making jokes about obesity and worry about becoming obese themselves. Hacking claims that the existence of such feedback shows that human kinds cannot be natural kinds.

J. Bogen ([1988]) has interpreted Hacking as claiming that human kinds are not natural kinds because the classification of human kinds results in feedback. Hacking rejects such an interpretation. In any case, as Bogen points out, such an argument would fail because our classificatory practices also result in feedback that alters some natural kinds. For example, because marijuana is classified as illegal the plants are grown in attics and wardrobes altering their physical appearance. As another example, the characteristics of domestic livestock change over time because particular animals are classified as being the 'Best in Show' and are used in selective breeding-sheep and pigs would now look very different if it weren't for our classificatory practices.

Hacking's argument that human kinds are not natural kinds must rest, not merely on the fact that feedback occurs, but rather on the fact that it occurs in a particular way. The difference, Hacking claims ([1997], p.15), is that feedback in human kinds occurs because subjects become aware of the ways in which they are being described and judged.

This idea needs working on before it can become an argument that human kinds cannot be natural kinds. As it stands Hacking has merely claimed that human kinds can be affected by a mechanism to which other kinds of entity are immune. Although this shows that there is some difference between human kinds and other kinds, it is not sufficient to show that this difference is of any fundamental significance. After all many other types of entity can be affected by mechanisms to which only entities of that type are vulnerable. While it is true that only human kinds are affected by the subjects' ideas, it is also true that only bacteria are affected by antibiotics, and that only domestic animals can be selectively bred. But no one would cite this as evidence that 'bacterial kinds' or 'domestic animal kinds' are not natural kinds.

The fact that only human kinds are affected by the subject's ideas will only be a reason for thinking that human kinds are distinct from natural kinds if an extra premise is added to the effect that being affected by ideas is of greater metaphysical significance than being affected by, say, antibiotics. In places Hacking suggests that feedback caused by the subject's awareness of being 
classified is important because it results in feedback occurring at a faster rate than that which affects natural kinds (see, for example, Hacking [1992], p.190). The thought seems to be that the speed with which change occurs confounds our attempts to use human kinds in inductive inferences. Such a claim is questionable. Do human kinds really change more quickly than bacteria and viruses mutate? In any case, a difference in the rate of feedback is inadequate to mark a fundamental metaphysical distinction between human kinds and natural kinds. If it were true that the characteristics of human kinds shifted more rapidly this would be reason, not for claiming that human kinds cannot be natural kinds, but rather for claiming that human kinds are not particularly useful natural kinds.

Alternatively, idea-dependence might be thought to matter because it betrays the subjective nature of a kind. The argument then would be that while natural kinds are objective, human kinds are affected by ideas and so subjective, and that thus human kinds cannot be natural kinds. Hacking gives no indication that this is a route he would wish to go down; however it is the most obvious option for someone who wishes to claim that idea-dependence is metaphysically significant and so worth pursuing here.

However, entities can be idea-dependent in two fundamentally different senses. And, as I will argue, idea-dependence in only one of these senses is indicative of subjectivity. Compare two senses in which ideas of female beauty 'affect' entities: In one case a woman, influenced by images of the 'ideal female form', decides she is too fat and so slims. Her altered shape is idea-dependent in the sense that her ideas concerning her weight caused her to slim. The development of Concord was dependent on the ideas of its developers in much the same kind of way; the developers had ideas about aeroplane designs, and these ideas feature in the causal history that culminated in the building of Concorde. Nevertheless, despite being in a sense 'idea-dependent' the reduction in the woman's weight, and the building of Concorde, are both perfectly objective. Idea dependence of this type results in objective changes in entities and is perfectly compatible with a kind being objective.

On the other hand consider the case where we look at old photos of the first Miss World. Miss World looks rather plump and short by today's standards, nevertheless presumably at the time she looked fine. Miss World's looks are also idea-dependent, but this time nothing about the photo of Miss World has actually changed. Rather the ideas prevalent in popular culture have made the properties of the photo appear different solely by acting on the viewers. The change is a relational 
change only. Such relational changes indicate that a kind, such as 'attractive women' is merely a subjective kind and so not a natural kind.

Hacking has shown that human kinds are idea-dependent. In order to show that this means that human kinds are subjective and thus cannot be natural kinds, it would need to be shown that human kinds are idea-dependent in the way that produces relational as opposed to genuine changes. All Hacking's examples, however, seem to be of cases where ideas produce genuine changes in peoples' behaviour. Take, for example, the case of Multiple Personality Disorder (Hacking, [1995a]). When patients with personalities of the opposite sex and animal personalities started to appear on American chat shows and be written about in magazines more and more patients started presenting with similar symptoms. The ideas prevalent in popular culture affected the symptoms typical of Multiple Personality Disorder. Still, here it seems that the ideas about Multiple Personality Disorder caused a genuine change in patients' symptoms. Patients really did start barking as a result of ideas. Such a claim need no more incriminate the kind 'Multiple Personality Disorder' than the claim that changing views on animal welfare have resulted in fewer dogs having their tails docked incriminates the kind 'dog'. In order to show that the changes in the symptoms of Multiple Personality Disorder indicate that it is not a natural kind, Hacking would need to show that barking, like beauty, is in the eye of the beholder, and he makes no suggestion that this is the case.

\subsection{Conceptual Feedback}

Hacking's argument for feedback at a conceptual level is dependent on Elizabeth Anscombe's account of intentional action. In her 1957 monograph, Intention, Anscombe considers the circumstances under which an action can be said to be intentional. Her solution is that an action $\mathrm{X}$ can be said to be intentional when the actor could respond to the question 'Why are you doing X?' by giving a reason for acting. If the actor cannot answer the 'Why?' question because he is not aware that he is doing $\mathrm{X}$, or if he knows he is X-ing but only because he has observed it, e.g. blushing, or if the actor knows he is X-ing but the cause is presumed to be purely non-mental, e.g. a muscle spasm, then the behaviour is not an intentional action.

On such an account an action is only intentional under a description because occasionally when we ask an agent 'Why are you X-ing?' he may fail to recognise his action under certain 
descriptions. For example, I am in the kitchen X-ing, where X may be either 'cooking' or 'getting in the way of my flat-mates'. I recognise my action only under the description of 'cooking', as I have not noticed that I am getting in the way. The action passes The Why Test, and thus is an intentional action, only under the description of 'cooking'.

Following Anscombe, Hacking uses the slogan 'intentional actions are actions under a description' in his argument that feedback occurs in human kinds:

1. Intentional actions are actions under a description.

2. Intentional actions make us the kind of person we are.

New descriptions allow new intentional actions, which allow new kinds of person.

If Hacking is correct then the creation of new descriptions makes logically possible the [3]

creation of new kinds of person. In creating new terminology the human sciences would make it possible for people to act in new ways. Here, however, I shall argue that Hacking's argument fails because he has misinterpreted Anscombe's phrase 'under a description'.

The phrase 'under a description' occurs throughout Anscombe's monograph. However its use is idiosyncratic and in 'Under a Description' Anscombe explains how she intended the phrase. She writes, 'under a description is "qua"...in modern dress'. Anscombe gives an example indicating that she uses 'qua' in the usual manner, she writes 'A may, qua B, receive such-and-such a salary and, qua C, such-and-such a salary.' ([1971], p.208).

If Anscombe in fact meant 'Intentional action is only intentional qua some aspect' why did she use the misleading phrase 'under a description'? Anscombe worked in the ordinary language tradition. Her monograph aims to give an account of what we say about commonplace actions. Within Anscombe's domain of the everyday the possibility of something being intended qua X, where there is no description that refers to $\mathrm{X}$ does not have to be considered, as it is fair to assume that all commonplace intentions have already been described. Thus Anscombe can treat 'under a description' and 'qua' as equivalent.

Hacking, however, is interested precisely in the situations that Anscombe can ignore. Hacking wants to consider the new possibilities for action created by a new description; he needs to contrast what was possible before the description was invented with what is possible after. In such 
cases the interpretation of 'under a description' becomes key. Consider Ug the caveman, sitting in his cave at the dawn of time before language developed. According to Hacking, Ug cannot intentionally light a fire, go outside, or hum himself a tune-as there are no descriptions, Ug must wait for them to develop before he can intentionally do anything.

If, on the other hand, we take 'under a description' to merely mean 'qua', Ug is free to intentionally act in many ways. Ug can intend his banging flints qua a way to make a fire, rather than qua a way to make a noise. Although we cannot use The Why Test to find out what Ug intends to do, there are other ways in which we can decide what it is that he intends. We can consider Ug's probable motives: if it is cold he would have reason to make a fire, if other people are banging drums he probably wants to make a noise. We can watch Ug's response when we intervene in his action-if he intends to light a fire bringing in wood will tend to make him smile, if he's starting a music session singing would probably be more welcome.

Such an approach fits in well with Anscombe's discussion of the intentional actions of nonverbal agents. In 'Under a Description' she discusses a bird who lands on a twig that happens to be both covered in bird lime and near some seeds. The bird, she says, lands on the twig with the intention of reaching a seed but not with the intention of landing in the bird lime. We infer the bird's intention by attributing intentions that are appropriate for the bird given its perceptual apparatus, its intelligence, and typical bird behaviour. We think that birds can identify seeds, that they get hungry, and that typically birds try to get seeds, and so we attribute the intention of getting the seed to the bird.

The problem of deciding how an action is intended arises because one bodily behaviour can help fulfil several different possible goals. Thus we cannot decide what someone intends merely by looking at their movements. Hacking presumes that the conditions under which an intentional action can be performed are identical to the conditions under which an observer can infer the actor's intentions. He sets about asking when intentional actions are possible via asking how an observer can determine what it is that an agent intends, and assumes that if one cannot tell what an agent intends that no intentional action is possible. This is only permissible if some verificationist principle is adopted. Even if such a principle is considered acceptable, however, if 'under a description' is interpreted as 'qua' there is no reason to think that intentional actions are logically dependent on the existence of descriptions. Asking an actor to explain his actions is one way, but not the only way, to 
discover what an agent intended. Using the method of asking the actor requires descriptions, but as there are other means of inferring an actor's intentions which do not depend on descriptions, it cannot be concluded that descriptions are essential for intentional actions. Ug can intend to make a fire, and the bird can intend to land on the twig, without any descriptions being required. In such cases Hacking is simply wrong to claim that descriptions are required for intentional action.

Of course not all actions are so contingently linked to language. Consider the act of marrying [4]

someone, or the act of promising. In order to marry a man one actually has to say, 'I hereby take this man to be my lawfully wedded husband'. Similarly, someone can only promise to do something if they say, 'I promise to do X'. Without the descriptions relating to marriage and promising, there can be no such actions. I suggest, however, that such actions form an unusual class. Such actions are peculiar in that they are defined in pseudo-legal ways, and the law, of course, unlike everyday thinking, dislikes ambiguity. It is extremely important to people that they have a way of being sure whether or not they are married, and of being sure when they have been promised something. That these actions are defined as being tied to the utterance of descriptions acts to reduce possible sources of doubt as to whether an intentional action has occurred or not. That one actually has to say particular sentences in order to get married makes it extremely unlikely that one could find oneself considered married by accident. In contrast, in everyday life we are able, and forced, to tolerate uncertainty, and accept conventions whereby we can say that this or that person intended to do X or $\mathrm{Y}$ even though there is a chance that we are wrong. In short while there is a class of pseudo-legal actions that are logically tied to their descriptions, such actions are only a sub-set of all actions. I can accept that the logical link between such actions and descriptions means that kinds such as 'promisee' and 'husband' will not form natural kinds, but still argue that no such logical link between actions and descriptions affects kinds such as 'autistic person', 'obese person' and 'homosexual'.

I accept, in addition, that there might be contingent links between descriptions and the ability to perform certain types of intentional actions. Some actions might be too complicated to perform without the aid of a description, for example, cooking certain complicated dishes might require a recipe describing what is to be done at each stage. It might also be true that actors only act in certain ways because certain descriptions exist in a culture, for example, it might be true that the existence of a tradition of limerick writing in a sense makes it possible for us to intend to write a limerick, as 
without the tradition no individual would ever think of doing such a strange thing. In such cases, however, our ability to perform certain intentional actions is only contingently dependent on the existence of certain descriptions. The descriptions in the culture may cause us to act in certain ways, but they are not needed for it to be logically possible for us to act in certain ways. Hacking's conceptual feedback has collapsed back into his cultural feedback and, as I have already argued, the existence of such feedback does not show that human kinds are not natural kinds. I conclude that Hacking's argument fails and he has not shown that human kinds are not natural kinds.

Critics may protest that even if I am right this is not important. Fair enough, they may say, Hacking was mistaken in claiming to have shown that human kinds cannot be natural kinds. Still, his important claims, which have to do with feedback and interaction, remain. Through his case studies concerning Multiple Personality Disorder, child abuse, and autism, Hacking has shown us that kinds of people emerge along with our categorisations of them. People are affected by categories, and categories by people, and the important thing Hacking has done is to draw our attention to this. The arguments that human kinds are not natural kinds, they may suggest, are not really that important to this project.

I am happy to accept that many useful things can be learned from Hacking's case studies even if my argument in this paper is correct. This paper is not intended to be a generalised exercise in Hacking-bashing, and I am happy for Hacking to be right about a great many things. My sole claim in this paper is that Hacking has failed to show that human kinds are not natural kinds. Still, I hold that this claim is important. In the literature on natural kinds, natural kinds are often thought to appear in natural laws, to function in explanations, and to support inductive inferences. As such, whether human kinds are distinct from natural kinds matters. If human kinds are natural kinds then this suggests that accounts of laws, explanations, and the basis of sound inductive inferences, developed for the natural sciences, can be carried across in to the human sciences. If human kinds are not natural kinds, then this will be a reason for thinking that distinct accounts will be required.

Of course, even if I am right and Hacking's argument fails, there may be other arguments not considered here that do succeed in showing that human kinds are not natural kinds. This paper is not intended to settle once and for all the question of whether human kinds are natural kinds, it merely seeks to show that one influential argument against this claim fails. 
Acknowledgements

I would like to thank John Forrester, Ian Hacking, Martin Kusch, Peter Lipton, and Tony Marcel for comments on drafts of this paper. A version of this paper was presented a conference of the European Society for Philosophy and Psychology and I am grateful for the comments of those present.

\section{References}

Anscombe, E. [1957]: Intention, Oxford: Basil Blackwell.

Anscombe, E. [1971]: 'Under a Description', in E. Anscombe, 1981, Metaphysics and the Philosophy of Mind, Oxford: Basil Blackwell, pp.208-219.

Bird, A. [1998]: Philosophy of Science, London: U.C.L. Press.

Bogen, J. [1988]: 'Comments', Nous, 22, pp.65-6.

Boyd, R. [1991]: 'Realism, Anti-foundationalism and the Enthusiasm for Natural Kinds', Philosophical Studies, 61, pp.127-148.

Dupré, J. [1981]: 'Natural Kinds and Biological Taxa', The Philosophical Review, XC, pp.66-90.

Dupré, J. [1993]: The Disorder of Things, New Haven: Yale University.

Ghiselin, M. [1974]: ‘A Radical Solution to the Species Problem', Systematic Zoology, 23, pp.53644. 
Hacking, I. [1986]: 'Making Up People', in T. Heller, M. Sosna and D. Wellberry (eds.), 1986, Reconstructing Individualism, Stanford, California: Stanford University Press, pp.222-236.

Hacking, I. [1988]: 'The Sociology of Knowledge About Child Abuse', Nous, 22, pp. 53-63.

Hacking, I. [1990]: 'Natural Kinds', in R. Barrett and R. Gibson (eds.), 1990, Perspectives on Quine, Oxford: Blackwell, pp.129-143.

Hacking, I. [1991]: ‘A Tradition of Natural Kinds’, Philosophical Studies, 91, pp.109-126.

Hacking, I. [1992]: ‘World-Making by Kind-Making: Child Abuse For Example’, M. Douglas and D. Hull (eds.), 1992, How Classification Works, Edinburgh: Edinburgh University Press, pp.180238 .

Hacking, I. [1993]: 'Working in a New World: The Taxonomic Solution', in P. Horwich (ed.), 1993, World Changes, Massachusetts: Massachusetts Institute of Technology, pp. 275-310.

Hacking, I. [1995a]: Rewriting the Soul, Princeton: Princeton University Press.

Hacking, I. [1995b] 'The Looping Effects of Human Kinds', in D. Sperber and A. Premark (eds.), 1995, Causal Cognition, Oxford: Clarendon Press, pp.351-394.

Hacking, I. [2000]: The Social Construction of What?, Cambridge Massachusetts: Harvard University Press.

Hull, D. [1976]: ‘Are Species Really Individuals?’, Systematic Zoology, 25, pp.174-91.

Millikan, R. [1997]: 'On Cognitive Luck: Externalism in an Evolutionary Frame’, in M. Carrier and P. Machamer (eds.), 1997, Mindscapes: Philosophy, Science and the Mind, Pittsburgh: University of 
Pittsburgh Press, pp.207-219.

Mill, J.S. [1973]: Collected Works of John Stuart Mill. J. Robson (ed.) London: Routledge.

Nagel, E. [1979]: The Structure of Science, Indianapolis: Hackett Publishing Company.

Quine, W. [1969]: 'Natural kinds', in Ontological Relativity and Other Essays, 1969, New York: Columbia University Press, pp.114-38.

Wilkerson, T. [1995]: Natural Kinds, Aldershot: Aldbury. 
[1]

Classic formulations of the links between kinds, laws, explanations, and inductive inferences can be found in Quine ([1969]) and Nagel ([1979], pp.30-1, footnote 2). For more recent discussion of these links see Bird ([1998]).

[2]

Indeed, by 1999, in The Social Construction of What?, Hacking abandons all talk of natural kinds as 'Too much philosophy has been built into the epithet "natural kind"'(p.105 ). In this work he uses the term 'indifferent kind' instead. It is true that Hacking has written several papers on natural kinds, but these papers assess the importance of natural kinds in other thinkers' thought, rather than putting forward Hacking's own views on the nature of natural kinds. Hacking ([1990]) clarifies Quine's account of natural kinds; Hacking ([1993]) explains how natural kinds can be used to make sense of Kuhn's claim that the world changes after a paradigm shift; Hacking ([1991]) traces the history of the tradition of natural kinds in Western philosophy.

[3] [4]

That Hacking's claim concerns logical possibility comes out most clearly in Hacking ([1986]).

I am grateful to Martin Kusch for these examples. 